\title{
Lympho̊dem
}

\section{Früh diagnostizieren, vernetzt versorgen}

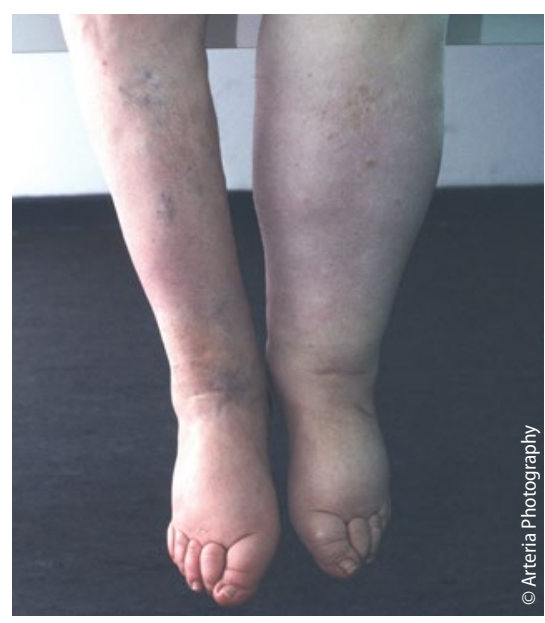

Von einer Beckenvenenthrombose ausgelöstes Lymphödem.

Experten gehen von über einer Million Patienten mit Lymphödemen in Deutschland aus. Viele von ihnen haben eine jahrelange Odyssee hinter sich, bis die Erkrankung korrekt diagnostiziert wird. Dabei sei die Diagnose gar nicht so schwierig zu stellen, betonte die Präsidentin der Deutschen Gesellschaft für Lymphologie, Dr. Anya Miller.

\section{Erster Schritt: die Ursachensuche}

Wichtig sei als erster Schritt der Ausschluss systemischer Ödemursachen. $\mathrm{Zu}$ diesen gehören Herzinsuffizienz, Nierenversagen und Lebersynthesestörungen.

Im zweiten Schritt sollten dann mögliche phlebologische Ursachen einer Ödementwicklung abgeklärt werden. Dies ist in der Regel per Ultraschall möglich. Bringt auch dieser Schritt keine Klarheit, bleiben in erster Linie Lipödem und Lymphödem als mögliche Diagnosen übrig.

Prof. Knut Kröger, Krefeld, betonte in diesem Zusammenhang die Bedeutung des Stemmer-Zeichens. Dabei wird die Haut über dem Grundgelenk der zweiten Zehe mit Daumen und Zeigefinger angehoben. Gelingt das nicht, spricht das für ein Lymphödem, da dieses die Zehen meist mit einbezieht. Das Lipödem hingegen beschränkt sich in der Regel auf den Bereich oberhalb des Sprunggelenks, und das Phlebödem endet zumindest in frühen Stadien am Fußrücken.

\section{Therapie der Wahl: die komplexe Entstauung}

Mit einer komplexen Entstauungstherapie (KPE) kann die Lebensqualität der Patienten verbessert und das Ödemvolumen reduziert werden [Lasinski BB et al. PM\&R. 2012;4:580-601]. Ein langfristiger Erfolg stelle sich aber nur ein, wenn die manuelle Lymphdrainage durch Kompressionstherapie, Bewegungstherapie und Hautpflege ergänzt werde, betonte Miller. Auch sei eine hohe Adhärenz des Patienten insbesondere in Bezug auf Kompression und Hautpflege wichtig.

Erfolgreich umsetzen lasse sich die $\mathrm{KPE}$ in erster Linie in regionalen lymphologischen Netzwerken, von denen es deutschlandweit rund 70 gibt. „Ärzte, die Lymphödempatienten versorgen wollen, sollten sich einem Netzwerk anschließen“, sagte Prof. Gerd Lulay vom Gesundheitszentrum Mathias-Spital
Rheine. Die Netzwerke gewährleisteten zum einen Qualitätsstandards bei Physiotherapie und Sanitätshäusern. Zum anderen sei es im Netzwerk einfacher, budgetierte Maßnahmen zu verordnen, ohne deswegen in Schwierigkeiten mit Kassen oder KVen zu kommen.

Philipp Grätzel

- Expertenworkshop Lymphologie; Berlin, Januar 2017 (Veranstaler: Springer Medizin, Medical Data Institute)

\section{Neue Fixkombination Candesartan/Amlodipin verfügbar}

Seit Mitte November 2016 steht die Fixkombination CandeAmlo Hexal ${ }^{\circledR}$ für Patienten zur Verfügung, deren Blutdruck mit der gleichzeitigen Gabe von Candesartan und Amlodipin im selben Dosierungsbereich adäquat kontrolliert wird. Fixkombinationen werden heute für die Hochdrucktherapie in den Leitlinien internationaler Fachgesellschaften empfohlen, weil sie geeignet sind, die bei Bluthochdruck generell niedrige Therapietreue zu verbessern.

Das Präparat wird zur einmal täglichen Einnahme in Form von Hartkapseln mit den Wirkstärken $8 / 5 \mathrm{mg}, 8 / 10 \mathrm{mg}, 16 / 5 \mathrm{mg}$ und $16 / 10 \mathrm{mg}$ angeboten. Durch die preisliche Stellung von CandeAmlo Hexal ${ }^{\circledR}$ reduziert sich - wenn man von einer identischen Reichweite der verordneten Arzneimittel ausgeht - die Zuzahlung der Patienten von bisher 10 auf zukünftig 5 Euro pro Verordnung.

- Nach Informationen von Hexal 